\title{
Scalar curvature and uniruledness on projective manifolds
}

\author{
Gordon Heier And Bun Wong
}

\begin{abstract}
It is a basic tenet in complex geometry that negative curvature corresponds, in a suitable sense, to the absence of rational curves on, say, a complex projective manifold, while positive curvature corresponds to the abundance of rational curves. In this spirit, we prove in this note that a projective manifold $M$ with a Kähler metric with positive total scalar curvature is uniruled, which is equivalent to every point of $M$ being contained in a rational curve. We also prove that if $M$ possesses a Kähler metric of total scalar curvature equal to zero, then either $M$ is uniruled or its canonical line bundle is torsion. The proof of the latter theorem is partially based on the observation that if $M$ is not uniruled, then the total scalar curvatures of all Kähler metrics on $M$ must have the same sign, which is either zero or negative.
\end{abstract}

\section{Introduction and statement of the results}

A complex projective manifold $M$ of dimension $n$ is uniruled if and only if there exists a dominant rational map from $\mathbb{P}^{1} \times N$ onto $M$, where $N$ is a complex projective variety of dimension $n-1$. Equivalently, $M$ is uniruled if and only if there exists a rational curve passing through every point of $M$. It is a well-known open problem whether all complex projective manifolds of Kodaira dimension $-\infty$ are uniruled. In dimensions one and two, this is classical. In dimension three, this follows from the minimal model program in algebraic geometry. The converse of this statement is easily verified in any dimension.

From a differential geometric point of view, complex projective manifolds of Kodaira dimension $-\infty$ resemble compact Kähler manifolds of positive scalar curvature. Yau [16] proved that the Kodaira dimension of a compact Kähler manifold with positive total scalar curvature must be $-\infty$. Moreover, it is not hard to see that every uniruled manifold is rationally dominated by a Kähler manifold with positive scalar curvature (see Section 5). Nonetheless, the converse of this statement is difficult to establish without a good method 
to guarantee the existence of rational curves under the assumption of positivity of scalar curvature.

One highlight of the development of this type of problem along differential geometric lines was when Siu-Yau [15] proved the Frankel conjecture, which states that a compact Kähler manifold of positive bisectional curvature is biholomorphic to a projective space. The crucial step of their proof relied on the production of a rational curve using the analyticity of a stable harmonic map under the positive bisectional curvature assumption. Around the same time, Mori [12] settled a more general conjecture of Hartshorne, which states that a compact complex manifold with ample tangent bundle is biholomorphic to projective space. In his proof, Mori introduced the bend and break technique, which is a method, partially relying on arguments in positive characteristic, to show the existence of rational curves based on certain positivity assumptions. Mori's technique has important consequences. For example, let $C$ be a curve passing through a point $x$ on a complex projective manifold $X$, and let $K$ denote the canonical line bundle of $X$. If the intersection number $-K . C$ is positive, then there is a rational curve passing through the same point $x$. In particular, a Fano manifold $X$, i.e., a compact complex manifold with positive first Chern class $c_{1}(X)=-c_{1}(K)$, is uniruled. Note that, due to Yau's proof of Calabi's conjecture, a compact complex manifold has positive first Chern class if and only if it possesses a Kähler metric with positive Ricci curvature. Actually, more is true, namely that Fano manifolds are rationally connected. We refrain from giving detailed attributions of these results and instead refer the reader to the monographs $[3,9]$ and the references contained therein.

The first main result proven in this note is that, instead of assuming positivity of the Ricci curvature, it suffices to make the much weaker assumption of positivity of the total scalar curvature to obtain uniruledness, as differential geometers would desire. Note that on the spectrum of the usual positivity notions in differential geometry, positive total scalar curvature, which is expressed in terms of the positivity of just one number, is the weakest notion of positivity. Our result is the following.

Theorem 1.1. Let $M$ be a projective manifold with a Kähler metric with positive total scalar curvature. Then $M$ is uniruled.

The general philosophy is that a complex projective manifold which is not uniruled must satisfy certain generic positivity conditions in terms of its cotangent bundle in light of $[10,11]$. Concretely, we will be using the result of Boucksom-Demailly-Paun-Peternell [1], who proved that if a projective manifold is not uniruled then it has a pseudo-effective canonical 
line bundle. On the whole, our results should be considered as differential geometric interpretations of the theory of bend and break and the recent work [1] on the duality of certain cones (see [1, Th. 2.2], reproduced below as Theorem 5.1).

In general, it is not true that a Kähler manifold with positive holomorphic sectional curvature has positive first Chern class (see the examples on Hirzebruch surfaces constructed by Hitchin in [5]). It is thus not possible to directly conclude the uniruledness of a Kähler manifold with positive holomorphic sectional curvature from the earlier results. However, it does follow from a pointwise argument due to Berger [2] that the scalar curvature (and thus also the total scalar curvature) of a Kähler metric of positive holomorphic sectional curvature is also positive. Thus, we obtain the following corollary of Theorem 1.1.

Corollary 1.1. Let $M$ be a projective manifold with a Kähler metric with positive holomorphic sectional curvature. Then $M$ is uniruled.

Remark 1.1. It was proven in [6] in dimension 3 that a Kähler manifold with negative holomorphic sectional curvature has negative first Chern class. This is conjectured to be true even in arbitrary dimension and represents a significant distinction between the cases of positive and negative curvature.

Our second main result is a characterization of projective manifolds with zero total scalar curvature.

Theorem 1.2. Let $M$ be a projective manifold with a Kähler metric with total scalar curvature equal to zero. Then either $M$ is uniruled or the canonical line bundle of $M$ is a torsion line bundle.

A key ingredient in the proof of Theorem 1.2 will be the following statement on the uniqueness of the sign of the total scalar curvature in the absence of uniruledness. It appears to be new and interesting in its own right, so we state it as another theorem.

Theorem 1.3. Let $M$ be a projective manifold which is not uniruled. Then the total scalar curvatures of all Kähler metrics on $M$ must have the same sign, which is either zero or negative.

In this paper, we take a Calabi-Yau manifold to be a compact complex manifold admitting a Ricci-flat Kähler metric. For a projective manifold $X$, using the results in $[7,13,17]$, one can easily show that being Calabi-Yau 
is equivalent to the canonical line bundle of $X$ being a torsion line bundle. Thus, the conclusion of Theorem 1.2 can be restated as saying that either $M$ is uniruled or Calabi-Yau.

We observe that, in the uniruled case, the sign of the (total) scalar curvature may be arbitrary. The simplest example is that of $\mathbb{P}^{1} \times N$, where $N$ is a compact hyperbolic Riemann surface and both manifolds come with their standard constant scalar curvature Kähler metrics. After multiplying one of the factors in the product metric with an appropriate positive number, the scalar curvature can be made to have an arbitrary sign.

We also state the following theorem, which is stronger than Theorem 1.3. It simply follows from combining the statements of Theorems 1.2 and 1.3, but it may be of particular interest to readers focused on complex differential geometry.

Theorem 1.4. Let $M$ be a projective manifold which is not uniruled. Then either all Kähler metrics on $M$ have negative total scalar curvature or $M$ is Calabi-Yau.

Since a compact Kähler manifold with negative holomorphic sectional curvature cannot be Calabi-Yau due to [6, Th. 2.3] and does not admit any rational curves, the following corollary is immediate from Theorem 1.4.

Corollary 1.2. Let $M$ be a projective manifold with a Kähler metric with negative holomorphic sectional curvature. Then the total scalar curvature of any Kähler metric on $M$ is negative.

Finally, we would like to point out that it is commonly expected that a projective manifold with pseudo-effective canonical line bundle has non-negative Kodaira dimension (see $[1$, p. 1]). If a proof of this open problem were known, most of our results would become easy to prove using the method in [16] relating scalar curvature and the Poincaré-Lelong formula (see page 760). Namely, due to Boucksom et al. [1], non-uniruledness could then be directly translated to the existence of a non-zero pluricanonical section. For a very recent development concerning such problems of abundance conjecture-type, we refer to the manuscript [14] by Siu.

\section{The case of positive total scalar curvature}

In this section, we prove Theorem 1.1. We begin by first recalling some standard notions and notations (see $[1,3,4]$ ). 
A current $T$ of bidegree $(1,1)$ on a complex manifold of dimension $n$ is positive if for every choice of smooth $(1,0)$ forms $a_{1}, \ldots, a_{n-1}$, the distribution

$$
\sqrt{-1}^{n-1} T \wedge a_{1} \wedge \bar{a}_{1} \wedge \cdots \wedge a_{n-1} \wedge \bar{a}_{n-1}
$$

is a positive measure.

A line bundle $F$ on a projective manifold $X$ is pseudo-effective if its first Chern class $c_{1}(F)$ is in the closed cone in $H^{1,1}(X)$ generated by the classes of effective divisors. For our purposes, we think of pseudo-effective line bundles in terms of an equivalent differential-geometric characterization: a line bundle $F$ on a projective manifold $X$ is pseudo-effective if it carries a singular hermitian metric $h$, locally given by $h=\mathrm{e}^{-\varphi}$ with $\varphi \in L_{\text {loc }}^{1}$, such that its (globally well-defined) curvature current

$$
\Theta(h)=\sqrt{-1} \partial \bar{\partial} \varphi
$$

is a positive current of bidegree $(1,1)$.

We note also that, under the canonical isomorphism mapping the usual Dolbeault cohomology with coefficients in $F$ to the corresponding cohomology for currents, the class of $\Theta(h)$ corresponds to the class $2 \pi c_{1}(F)$, where $c_{1}(F)$ is the first Chern class of $F$.

Furthermore, for a Kähler metric $g=\sum_{i, j=1}^{n} g_{i j} d z_{i} \otimes d \bar{z}_{j}$, its Ricci curvature form is given by

$$
\operatorname{Ric}(g)=-\sqrt{-1} \partial \bar{\partial} \log \operatorname{det}\left(g_{i \bar{j}}\right)
$$

By a result of Chern, the class of the form $\frac{1}{2 \pi} \operatorname{Ric}(g)$ is equal to $c_{1}(M)=$ $c_{1}(-K)$, where $K$ is the canonical line bundle of $M$. The scalar curvature $s$ of $g$ is defined to be the trace of $-\sqrt{-1} \operatorname{Ric}(g)$ with respect to a unitary frame. Finally, the total scalar curvature of $g$ is defined to be

$$
\int_{M} s \frac{\omega^{n}}{n !},
$$

where $\omega=\frac{\sqrt{-1}}{2} \sum_{i, j=1}^{n} g_{i j} \mathrm{~d} z_{i} \wedge \mathrm{d} \bar{z}_{j}$ is the Kähler form associated to $g$.

To prove Theorem 1.1, let $g$ now denote the Kähler metric on $M$ with positive total scalar curvature. It follows from linear algebra and the definition of scalar curvature that

$$
\operatorname{Ric}(g) \wedge \omega^{n-1}=\frac{2}{n} s \omega^{n}
$$

where $s$ is the scalar curvature of $g$. 
Now, assume that $M$ is not uniruled. By [1, Cor. 0.3], the canonical line bundle $K$ of $M$ is pseudo-effective, i.e., it carries a singular hermitian metric $h$ whose curvature current $\Theta(h)$ is a positive current of bidegree $(1,1)$. Since both $\Theta(h)$ and $-\operatorname{Ric}(g)$ represent $2 \pi c_{1}(K)$, we have

$$
\begin{aligned}
\int_{M} & \Theta(h) \wedge \omega^{n-1} \\
= & -\int_{M} \operatorname{Ric}(g) \wedge \omega^{n-1} \\
= & -\int_{M} \frac{2}{n} s \omega^{n} \\
& <0
\end{aligned}
$$

where the last inequality is due to the positivity of the total scalar curvature. On the other hand, the expression in (2.1) is non-negative, which yields a contradiction. The reason for the non-negativity is the positivity of $\Theta(h)$ and the fact that $\omega \wedge \cdots \wedge \omega$ can be written as a global sum of the form $\sum_{k=1}^{N} \sqrt{-1}^{n-1} a_{1}^{(k)} \wedge \bar{a}_{1}^{(k)} \wedge \cdots \wedge a_{n-1}^{(k)} \wedge \bar{a}_{n-1}^{(k)}$, where the $a_{i}^{(k)}, k=1, \ldots, N$, $i=1, \ldots, n-1$, are globally defined smooth $(1,0)$ forms. To justify this, we notice that every hermitian form can locally be represented in terms of a coframe of smooth $(1,0)$ forms $\varphi_{1}, \ldots, \varphi_{n}$ as a sum $\sqrt{-1} \sum_{k=1}^{n} \varphi_{k} \wedge \bar{\varphi}_{k}$. The globalization is then achieved by means of a partition of unity. For complete details, we refer the reader to the proofs of Lemmas 3.1 and 3.2, which contain identical arguments.

\section{Uniqueness of the curvature sign in the absence of uniruledness}

To prepare for the proof of Theorem 1.2 in the next section, we now prove Theorem 1.3. According to Theorem 1.1, positive total scalar curvature implies uniruledness. Thus, in order to derive a contradiction, we may assume that there are two Kähler metrics on $M$ such that one has zero total scalar curvature and one has negative total scalar curvature. We denote these Kähler metrics and their Kähler forms with $g_{0}, \omega_{0}$ and $g_{-}, \omega_{-}$, respectively. Since $M$ is compact, we can choose a sufficiently small positive number $\varepsilon$ such that $\omega=\omega_{0}-\varepsilon \omega_{-}$is still a Kähler form. Also, let $n=\operatorname{dim} M$, let

$$
A=\omega_{0}^{n-1},
$$


and

$$
B=\left(\varepsilon \omega_{-}\right)^{n-1}=\varepsilon^{n-1} \omega_{-}^{n-1} .
$$

Lemma 3.1. There exists a finite open covering $\left(U_{\nu}\right)$ of $M$ and positive integers $N_{\nu}$ such that for every $\nu$ and $k_{\nu} \in\left\{1, \ldots, N_{\nu}\right\}$, there exist smooth $(1,0)$ forms $a_{1}^{\left(\nu, k_{\nu}\right)}, \ldots, a_{n-1}^{\left(\nu, k_{\nu}\right)}$ on $U_{\nu}$ such that, on $U_{\nu}$, we have

$$
A-B=\sqrt{-1}^{n-1} \sum_{k_{\nu}=1}^{N_{\nu}} a_{1}^{\left(\nu, k_{\nu}\right)} \wedge \bar{a}_{1}^{\left(\nu, k_{\nu}\right)} \wedge \cdots \wedge a_{n-1}^{\left(\nu, k_{\nu}\right)} \wedge \bar{a}_{n-1}^{\left(\nu, k_{\nu}\right)} .
$$

Proof. In the case $n=2$, the statement of the lemma is true simply because $A-B=\omega_{0}-\varepsilon \omega_{-}$is a hermitian form. Namely, it is well known that on a small open set, there exists a coframe of smooth $(1,0)$ forms $\varphi_{1}, \ldots, \varphi_{n}$ such that

$$
A-B=\sqrt{-1} \sum_{k=1}^{n} \varphi_{k} \wedge \bar{\varphi}_{k}
$$

This proves the lemma in the case $n=2$. We now assume $n \geq 3$.

For real numbers $a, b$, it is a well-known fact that

$$
a^{n-1}-b^{n-1}=(a-b)\left(a^{n-2}+a^{n-3} b+\cdots+b^{n-2}\right) .
$$

Because $\omega_{0}, \omega_{-}$are each $(1,1)$ forms, we have

$$
\omega_{0} \wedge \omega_{-}=\omega_{-} \wedge \omega_{0},
$$

and the same identity thus holds in our situation:

$$
\begin{aligned}
A-B & =\omega_{0}^{n-1}-\left(\varepsilon \omega_{-}\right)^{n-1} \\
& =\left(\omega_{0}-\varepsilon \omega_{-}\right) \wedge\left(\omega_{0}^{n-2}+\left(\omega_{0}^{n-3}\right) \wedge\left(\varepsilon \omega_{-}\right)+\cdots+\left(\varepsilon \omega_{-}\right)^{n-2}\right) .
\end{aligned}
$$

Now note that $\omega_{0}-\varepsilon \omega_{-}, \omega_{0}, \omega_{-}$locally all have representations in terms of a coframe as on the right hand side of (3.1). Substituting these representations into the second line of the above display yields the expression whose existence is claimed in the lemma.

We continue with a proof of the following inequality.

Lemma 3.2. For $g_{0}$ and $A, B$ as above, the following holds.

$$
-\int_{M} \operatorname{Ric}\left(g_{0}\right) \wedge(A-B) \geq 0 .
$$


Proof. By Chern's result, $-\operatorname{Ric}\left(g_{0}\right)$ represents $2 \pi c_{1}(K)$. Due to the pseudoeffectivity of $K$, we have

$$
-\sqrt{-1}^{n-1} \int_{M} \operatorname{Ric}\left(g_{0}\right) \wedge a_{1} \wedge \bar{a}_{1} \wedge \cdots \wedge a_{n-1} \wedge \bar{a}_{n-1} \geq 0
$$

for smooth $(1,0)$ forms $a_{1}, \ldots, a_{n-1}$ on $M$.

Let $\left(\chi_{\nu}\right)$ be a partition of unity subordinate to the covering $U_{\nu}$. If $a_{1}^{\left(\nu, k_{\nu}\right)} \wedge \bar{a}_{1}^{\left(\nu, k_{\nu}\right)} \wedge \cdots \wedge a_{n-1}^{\left(\nu, k_{\nu}\right)} \wedge \bar{a}_{n-1}^{\left(\nu, k_{\nu}\right)}$ is one of the forms arising in Lemma 3.1, we can turn it into a globally defined form simply by multiplying it with $\chi_{\nu}$. It is immediate that for all $\nu$ and $k_{\nu}$,

$$
-\sqrt{-1}^{n-1} \int_{M} \operatorname{Ric}\left(g_{0}\right) \wedge\left(\chi_{\nu} a_{1}^{\left(\nu, k_{\nu}\right)} \wedge \bar{a}_{1}^{\left(\nu, k_{\nu}\right)} \wedge \cdots \wedge a_{n-1}^{\left(\nu, k_{\nu}\right)} \wedge \bar{a}_{n-1}^{\left(\nu, k_{\nu}\right)}\right) \geq 0
$$

because on $U_{\nu}$, we can replace $a_{1}^{\left(\nu, k_{\nu}\right)}$ by $\sqrt{\chi_{\nu}} a_{1}^{\left(\nu, k_{\nu}\right)}$ and apply (3.3). Furthermore, by Lemma 3.1,

$$
\begin{gathered}
-\int_{M} \operatorname{Ric}\left(g_{0}\right) \wedge(A-B) \\
=-\int_{M} \operatorname{Ric}\left(g_{0}\right) \wedge\left(\sum_{\nu} \sqrt{-1}^{n-1} \chi_{\nu} \sum_{k_{\nu}=1}^{N_{\nu}} a_{1}^{\left(\nu, k_{\nu}\right)} \wedge \bar{a}_{1}^{\left(\nu, k_{\nu}\right)} \wedge \cdots \wedge a_{n-1}^{\left(\nu, k_{\nu}\right)} \wedge \bar{a}_{n-1}^{\left(\nu, k_{\nu}\right)}\right) \\
=\sum_{\nu} \sum_{k_{\nu}=1}^{N_{\nu}}-\sqrt{-1}^{n-1} \int_{M} \operatorname{Ric}\left(g_{0}\right) \wedge\left(\chi_{\nu} a_{1}^{\left(\nu, k_{\nu}\right)} \wedge \bar{a}_{1}^{\left(\nu, k_{\nu}\right)} \wedge \cdots \wedge a_{n-1}^{\left(\nu, k_{\nu}\right)} \wedge \bar{a}_{n-1}^{\left(\nu, k_{\nu}\right)}\right) .
\end{gathered}
$$

Due to (3.4), the summation in the last line of the above display only contains non-negative numbers, which concludes the proof.

To finish the proof of Theorem 1.3, we now obtain a contradiction as follows. Note that the last equality in the display below is due to the fact that $-\operatorname{Ric}\left(g_{-}\right)$also represents $2 \pi c_{1}(K)$, and that the inequality is due to Lemma 3.2.

$$
\begin{aligned}
0 & =\int_{M} \operatorname{Ric}\left(g_{0}\right) \wedge A \\
& \leq \int_{M} \operatorname{Ric}\left(g_{0}\right) \wedge B \\
& =\int_{M} \operatorname{Ric}\left(g_{-}\right) \wedge B .
\end{aligned}
$$

The value of the last line in the above display is a positive constant times the total scalar curvature of $g_{-}$, which yields the desired contradiction. 


\section{The case of zero total scalar curvature}

In this section, we conduct the proof of Theorem 1.2 by assuming that $M$ is not uniruled and then showing that the canonical line bundle of $M$ is torsion. To this end, we will use the following well-known lemma (see [8, Ch. I, $\S 4$, Prop. 3], [3, 3.8]) based on the Hodge index theorem.

Lemma 4.1. Let $X$ be a smooth complex projective variety of dimension $n$. Let $D$ be a divisor on $X$ such that $D \cdot H^{n-1}=0$ for all ample divisors $H$. Then $D$ is numerically trivial.

To apply the lemma, take an arbitrary ample divisor on $M$. By Kodaira's embedding theorem, $m H$ is very ample for a sufficiently large positive integer $m$. Let $g$ be the Kähler metric that is obtained by restriction of the Fubini-Study metric on projective space after the embedding of $M$ furnished by $m H$. If $\omega$ is the Kähler form associated to $g$, then

$$
\begin{aligned}
m^{n-1} K \cdot H^{n-1} & \\
& =-\frac{1}{2 \pi} \int_{M} \operatorname{Ric}(g) \wedge \omega^{n-1} \\
& =-\frac{1}{2 \pi} \int_{M} \frac{2}{n} s \omega^{n} \\
& =0
\end{aligned}
$$

where the last equality is due to the uniqueness statement in Theorem 1.3 and the assumed existence of some Kähler metric of total scalar curvature equal to zero. By Lemma 4.1, we can conclude that $K$ is numerically trivial.

Moreover, it is a result towards the abundance conjecture due to Kawamata [7] (in the case of minimal varieties) and Nakayama [13] (in the general case) that in this situation the Kodaira dimension of $M$ is equal to zero, i.e., there exists a positive integer $\ell$ such that $H^{0}(M, \ell K)$ has a non-zero element $\sigma$. Since $\ell K$ is also numerically trivial, $\operatorname{div}(\sigma)=\emptyset$, which implies that $\ell K$ is the trivial line bundle. In other words, $K$ is torsion, q.e.d.

We conclude this section by giving an alternative proof of Theorem 1.2 without using the Hodge index theorem in the case when $M$ is a four-fold. It is based on the following special case of the abundance conjecture from [1, Th. 9.8].

Theorem 4.1. Let $X$ be a smooth projective four-fold. If $K_{X}$ is pseudoeffective and if there is a strongly connecting family $\left(C_{t}\right)$ of curves such that $K_{X} \cdot C_{t}=0$, then $\operatorname{kod}(X)=0$. 
Recall from $\left[1\right.$, p. 2] that a strongly connecting family $\left(C_{t}\right)$ of curves is defined to be such that any two sufficiently general points can be joined by a chain of irreducible $C_{t}$ 's.

Let $g$ be the Kähler metric on $M$ induced by the Fubini-Study metric on the ambient projective space. Again, we denote by $\omega$ the corresponding Kähler form. The intersections of $M$ with codimension 3 planes in projective space yield a strongly connecting family $\left(C_{t}\right)$ of curves due to Bertini's theorem. To determine the intersection number of a curve $C_{t}$ with $K$, observe that, using again the fact that $-\frac{1}{2 \pi} \operatorname{Ric}(g)$ represents $c_{1}(K)$,

$$
K \cdot C_{t}=-\frac{1}{2 \pi} \int_{M} \operatorname{Ric}(g) \wedge \omega^{3} .
$$

However, the value of the right-hand side of the above display is, up to a non-zero multiplicative constant, the total scalar curvature of $g$. According to Theorem 1.3, there cannot exist two Kähler metrics with different total scalar curvature on $M$, so the value of the right-hand side is zero. Due to Theorem 4.1, $\operatorname{kod}(X)=0$. To conclude that $K$ is torsion, we can argue as follows (as done by Yau in [16]).

Let $\ell \in \mathbb{N}^{+}$such that there exists $\sigma \in H^{0}(M, \ell K) \backslash\{0\}$. Assume that $\operatorname{div}(\sigma)=D \neq \emptyset$. Then $\Theta=\sqrt{-1} \partial \bar{\partial} \log |\sigma|^{2}$ is a closed positive current such that $\Theta$ represents $2 \pi \ell c_{1}(K)$. According to the Poincaré-Lelong formula,

$$
\begin{aligned}
0 & <2 \pi \int_{D} \omega^{n-1} \\
& =\int_{M} \Theta \wedge \omega^{n-1} \\
& =-\ell \int_{M} \operatorname{Ric}(g) \wedge \omega^{n-1} \\
& =-\ell \int_{M} \frac{2}{n} s \omega^{n} \\
& =0
\end{aligned}
$$

Contradiction. Thus, $\operatorname{div}(\sigma)=\emptyset$ and $K$ is torsion.

\section{Concluding remarks}

As we remarked in the Introduction, Theorem 1.1 is almost an equivalence for the following reason. Take an arbitrary Kähler metric on a desingularization $\tilde{N}$ of the variety $N$ in the definition of uniruledness of $M$. Then $\mathbb{P}^{1} \times \tilde{N}$ carries a product Kähler metric of positive (total) scalar curvature simply 
because the contribution to the curvature from $\tilde{N}$ can be scaled down until positivity is reached due to the positivity of the Fubini-Study metric on $\mathbb{P}^{1}$. However, $\mathbb{P}^{1} \times \tilde{N}$ still rationally dominates $M$. It thus seems likely that $M$ itself carries a Kähler metric of positive total scalar curvature, although we know of no rigorous proof.

A different way of looking at the possibility of a converse of Theorem 1.1 is via [1]. Recall that their main technical result is the following, which, combined with [11], immediately implies the above-used [1, Cor. 0.3].

Theorem 5.1 [1, Th. 2.2]. Let $X$ be a complex projective manifold. Then a class $\alpha \in \mathrm{NS}_{\mathbb{R}}(X)$ is pseudo-effective if (and only if) it is in the dual cone of the cone $\operatorname{SME}(X)$ of strongly movable curves.

Now, if $M$ is uniruled, and thus $K$ is not pseudo-effective, Theorem 5.1 yields, by the definition of strongly movable curves [1, Def. $1.3(\mathrm{v})]$, that there exist a modification $\mu: \tilde{M} \rightarrow M$ and very ample divisor classes $\tilde{A}_{1}, \ldots, \tilde{A}_{n-1}$ on $\tilde{M}$ such that

$$
\int_{M} c_{1}(M) \wedge \mu_{*}\left(\tilde{A}_{1} \wedge \cdots \wedge \tilde{A}_{n-1}\right)>0
$$

It thus seems probable that a uniruled $M$ itself possesses a Kähler metric of positive total scalar curvature, although we again do not have a complete proof. For some further evidence concerning this, we refer to [5, Cor. 5.18] and [16, Prop. 1].

Moreover, we remark that Theorem 1.2 is an equivalence except in the uniruled case. Namely, if the canonical line bundle of $M$ is torsion, then the first real Chern class of $M$ is equal to zero. By Yau's solution of the Calabi conjecture [17], there is a Ricci-flat Kähler metric on $M$, which has zero (total) scalar curvature.

For the future, we think it might be interesting to explore implications of other forms of positive curvature in terms of the existence of rational curves. This is motivated to a significant extent by the pioneering work of Yau [16]. We hope to return to this subject in a subsequent paper.

Finally, we address a suggestion from one of the referees by introducing the cohomological invariant

$$
T(\omega)=\int_{M} c_{1}(M) \wedge \omega^{n-1}
$$


where $\omega$ is an arbitrary Kähler form on $M$. Note that $T(\omega)$ simply equals

$\frac{(n-1) !}{\pi}$ times the total scalar curvature of the associated Kähler metric $g$. With the help of $T$, our results can be summarized as follows.

1. If $T$ assumes a positive value somewhere, then $M$ is uniruled.

2. If $T$ assumes more than one sign from $\{+, 0,-\}$, then $M$ is uniruled.

3. If $M$ is not uniruled and $T$ assumes the value zero somewhere, then $T$ is identically equal to zero and $M$ is Calabi-Yau.

\section{Acknowledgments}

The first author was partially supported by the National Security Agency under Grant Number H98230-12-1-0235. The United States Government is authorized to reproduce and distribute reprints notwithstanding any copyright notation herein.

\section{References}

[1] S. Boucksom, J.-P. Demailly, M. Paun and P. Peternell, The pseudo-effective cone of a compact Kähler manifold and varieties of negative Kodaira dimension, 2004, current version available at www-fourier.ujf-grenoble.fr/ demailly/research.html, earlier version: arXiv:math/0405285v1.

[2] M. Berger, Sur les variétés d'Einstein compactes, in 'Comptes Rendus de la IIIe Réunion du Groupement des Mathématiciens d'Expression Latine (Namur, 1965)', Librairie Universitaire, Louvain, 1966, 35-55.

[3] O. Debarre, Higher-dimensional algebraic geometry, Universitext, Springer-Verlag, New York, 2001.

[4] J.-P. Demailly, Multiplier ideal sheaves and analytic methods in algebraic geometry, in 'School on vanishing theorems and effective results in algebraic geometry (Trieste, 2000)', ICTP Lect. Notes, 6, 1-148, Abdus Salam Int. Cent. Theoret. Phys., Trieste, 2001.

[5] N. Hitchin, On the curvature of rational surfaces, in 'Differential geometry', Proceedings of the Symposium in Pure Mathematics, vol. XXVII, Part 2 (Stanford University, Stanford, California, 1973), Amer. Math. Soc., Providence, RI, 1975, 65-80. 
[6] G. Heier, S. S. Y. Lu and B. Wong, On the canonical line bundle and negative holomorphic sectional curvature, Math. Res. Lett. 17(6) (2010), 1101-1110.

[7] Y. Kawamata, Minimal models and the Kodaira dimension of algebraic fiber spaces, J. Reine Angew. Math. 363 (1985), 1-46.

[8] S. Kleiman, Toward a numerical theory of ampleness, Ann. of Math. (2), 84 (1966), 293-344.

[9] J. Kollár, Rational curves on algebraic varieties, in 'Results in Mathematics and Related Areas', 3rd Ser.: A Series of Modern Surveys in Mathematics, 32, Springer-Verlag, Berlin, 1996.

[10] Y. Miyaoka, Deformations of a morphism along a foliation and applications, in 'Algebraic geometry, Bowdoin, 1985 (Brunswick, Maine, 1985)', Proceedings of the Symposium in Pure Mathematics, 46, Amer. Math. Soc., Providence, RI, 1987, 245-268.

[11] Y. Miyaoka and S. Mori, A numerical criterion for uniruledness, Ann. of Math. (2), 124(1) (1986), 65-69.

[12] S. Mori, Projective manifolds with ample tangent bundles, Ann. of Math. (2), 110(3) (1979), 593-606.

[13] N. Nakayama, Zariski-decomposition and abundance, MSJ Mem. Math. Soc. Japan, Tokyo, 14, 2004.

[14] Y. T. Siu, Abundance conjecture, arXiv:math.AG/0912.0576v3, 2010.

[15] Y. T. Siu and S. T. Yau, Compact Kähler manifolds of positive bisectional curvature, Invent. Math. 59(2) (1980), 189-204.

[16] S. T. Yau, On the curvature of compact Hermitian manifolds, Invent. Math. 25 (1974), 213-239.

[17] S. T. Yau, On the Ricci curvature of a compact Kähler manifold and the complex Monge-Ampère equation I, Comm. Pure Appl. Math. 31(3) (1978), 339-411. 
Department of Mathematics

UNIVERSITY OF HOUSTON

4800 Calhoun Road

Houston, TX 77204

USA

E-mail address: heier@math.uh.edu

Department of Mathematics

UC RIVERSIDE

900 University Avenue

RIVERSIDE, CA 92521

USA

E-mail address: wong@math.ucr.edu

Received March 1, 2012 